\title{
GSK3 controls axon growth via CLASP-mediated regulation of growth cone microtubules
}

\author{
Eun-Mi Hur, ${ }^{1}$ Saijilafu, ${ }^{1}$ Byoung Dae Lee, ${ }^{2,3,4}$ Seong-Jin Kim, ${ }^{1}$ Wen-Lin Xu, ${ }^{1}$ \\ and Feng-Quan Zhou ${ }^{1,5,6}$ \\ ${ }^{1}$ Department of Orthopedic Surgery, Johns Hopkins University School of Medicine, Baltimore, Maryland 21205, USA; \\ ${ }^{2}$ Neuroregeneration Program, Institute for Cell Engineering, Johns Hopkins University School of Medicine, Baltimore, \\ Maryland 21205, USA; ${ }^{3}$ Stem Cell Program, Institute for Cell Engineering, Johns Hopkins University School of Medicine, \\ Baltimore, Maryland 21205, USA; ${ }^{4}$ Department of Neurology, Johns Hopkins University School of Medicine, Baltimore, \\ Maryland 21205, USA; ${ }^{5}$ The Solomon H. Snyder Department of Neuroscience, Johns Hopkins University School of Medicine, \\ Baltimore, Maryland 21205, USA
}

\begin{abstract}
Suppression of glycogen synthase kinase 3 (GSK3) activity in neurons yields pleiotropic outcomes, causing both axon growth promotion and inhibition. Previous studies have suggested that specific GSK3 substrates, such as adenomatous polyposis coli (APC) and collapsin response mediator protein 2 (CRMP2), support axon growth by regulating the stability of axonal microtubules (MTs), but the substrate(s) and mechanisms conveying axon growth inhibition remain elusive. Here we show that CLIP (cytoplasmic linker protein)-associated protein (CLASP), originally identified as a MT plus end-binding protein, displays both plus end-binding and lattice-binding activities in nerve growth cones, and reveal that the two MT-binding activities regulate axon growth in an opposing manner: The lattice-binding activity mediates axon growth inhibition induced by suppression of GSK3 activity via preventing MT protrusion into the growth cone periphery, whereas the plus end-binding property supports axon extension via stabilizing the growing ends of axonal MTs. We propose a model in which CLASP transduces GSK3 activity levels to differentially control axon growth by coordinating the stability and configuration of growth cone MTs.
\end{abstract}

[Keywords: GSK3; developmental and regenerative axon growth; growth cone microtubules; microtubule plus end-tracking proteins]

Supplemental material is available for this article.

Received May 11, 2011; revised version accepted August 11, 2011.

Glycogen synthase kinase 3 (GSK3), a multifunctional serine/threonine kinase, is a key regulator of neurogenesis, polarization, neurite outgrowth, and plasticity in the nervous system (Hur and Zhou 2010). Consistent with the wide range of actions, perturbations in GSK3 signaling are associated with a number of psychiatric and neurodegenerative diseases, and GSK3 inhibitors are under development as potential treatments for various neurological disorders (Jope and Johnson 2004; Martinez 2008; Medina and Castro 2008; Phukan et al. 2010). Despite the growing appreciation of GSK3 as a tempting therapeutic target for several diseases, the plethora of basic and essential processes regulated by GSK3 raises concerns about selectivity and the possibility of deleterious side effects. Thus, elucidation of GSK3 signaling and identification of the

${ }^{6}$ Corresponding author.

E-mail fzhou4@jhmi.edu.

Article is online at http://www.genesdev.org/cgi/doi/10.1101/gad.17015911. physiological target(s) of GSK3 responsible for mediating a particular outcome are of paramount importance to understand how this multifunctional kinase regulates a diverse array of functions in a specific manner and to have a better control over GSK3 signaling.

GSK3 is a well-known regulator of axon growth (Hur and Zhou 2010), but the consequence of GSK3 inhibition appears to be controversial: Some studies suggest that suppression of GSK3 activity is required for the formation and elongation of axons (Zhou et al. 2004; Jiang et al. 2005; Yoshimura et al. 2005), while others show that GSK3 inhibition drastically prevents axon extension (Owen and Gordon-Weeks 2003). To reconcile the controversy, it has been suggested that the final outcome of GSK3 inhibition might be determined by the extent of GSK3 inhibition and the downstream substrates involved (Kim et al. 2006). For the axon growth-promoting effect of GSK3 inhibition, previous studies have identified specific substrates of GSK3, such as adenomatous polyposis coli (APC) and collapsin 
response mediator protein 2 (CRMP2), as important mediators and suggested a model in which these substrates bind to microtubules (MTs) upon GSK3 inhibition to control the stability and dynamics of axonal MTs (Zhou et al. 2004; Yoshimura et al. 2005). However, the molecular target(s) of GSK3 and the underlying mechanisms that convey the axon growth inhibitory signal still remain enigmatic.

Recently, considerable interest has been centered on a group of proteins called MT plus end-tracking proteins (+TIPs) that specifically accumulate at the plus ends of MTs. +TIPs include members of structurally unrelated protein families, such as end-binding proteins (EBs), APC, cytoplasmic linker proteins (CLIPs), and CLIP-associated proteins (CLASPs). Localization of +TIPs at the plus ends places them in a strategic position to control different aspects of MTs, such as polymerization, dynamics, and stability (Akhmanova and Steinmetz 2008), all of which are essential for the control of axon growth. Despite the intriguing connection, the roles of +TIPs in neuronal morphogenesis and axon growth remain largely unexplored. Among +TIPs, APC (Zhou et al. 2004) and CLIPs (Neukirchen and Bradke 2011) have been shown to promote the formation and/or elongation of an axon by stabilizing MTs. These functions of +TIPs are in line with the longstanding view that stabilization of MTs induces growth cone advancement and supports axon extension, whereas MT destabilization mediates growth cone retraction and inhibits axon growth (Galjart 2005; Siegrist and Doe 2007).

CLASPs are widely conserved +TIPs that stabilize MTs in fungi, plants, and animals (Galjart 2005; Bratman and Chang 2008). In mammals, CLASPs are represented by two closely related paralogs: ubiquitously expressed CLASP1 and the nervous system-enriched CLASP2 (Akhmanova et al. 2001). The role of CLASP in controlling MT dynamics and stability as well as its regulation by GSK3 have been well documented (Akhmanova et al. 2001; Wittmann and Waterman-Storer 2005; Watanabe et al. 2009; AlBassam et al. 2010), but the physiological consequences of such regulations, especially in the mammalian nervous system, are unknown. Interestingly, genetic evidence has placed Orbit/MAST, the Drosophila ortholog of CLASP, downstream from Slit to induce growth cone repulsion and inhibit axon growth (Lee et al. 2004). Given that MT stabilization is generally associated with axon growth promotion rather than inhibition, these results have raised an intriguing question of how CLASP, a +TIP that functions to stabilize MTs as APC and CLIPs do, can suppress axon growth (Galjart 2005; Siegrist and Doe 2007).

The present study reports an unexpected finding that CLASP functions to both promote and restrict axon growth, and suggests that the opposing roles of CLASP are rooted in its unique MT-binding activities: CLASP supports axon extension when it binds to MT plus ends, whereas it restricts axon growth when bound along MT lattices. Furthermore, we identify CLASP2 as a physiological substrate of GSK3 that conveys the axon growth inhibitory signal upon GSK3 inhibition, and show that this function of CLASP2 requires its lattice-binding, but not the plus end-binding, activity. Finally, we provide in vivo evidence for a role of CLASP during axon regeneration in the mature nervous system with the advent of a novel technique that enables acute regulation of gene expression in dorsal root ganglions (DRGs) of live animals. Our study suggests GSK3-CLASP signaling as an intrinsic regulator of axon growth and unveils important mechanistic insight into how GSK3 deploys its substrate to differentially control developmental and regenerative axon growth.

\section{Results}

CLASP2 knockdown induces marked and specific enhancement of axon growth in embryonic cortical neurons

To investigate a role of CLASP in the mammalian nervous system, we used embryonic cortical neurons, a widely employed system to study neuronal morphogenesis. In cortical neurons, we found that CLASP was enriched in the growth cones of the axon and dendrites as well as in the cell body (Fig. 1A). Using previously reported RNAi sequences (Mimori-Kiyosue et al. 2005), we then generated bicistronic plasmids encoding Venus (a variant of YFP) and an shRNA targeting either CLASP1 (shCLASP1) or CLASP2 (shCLASP2) and validated the efficiency in a neuronal cell line, CAD (Fig. 1B). Transfection of shCLASP2 but not shCLASP1 markedly promoted axon growth in cortical neurons when cells were cultured at a low density (Fig. 1C,D). Similarly, axon growth was enhanced by a pool of siRNAs targeting four different regions in CLASP2 (ON-TARGETplus siCLASP2 from Dharmacon, Inc.). The axon growth-promoting effect of CLASP2 depletion was also evident when cell density was increased, even though the average axon length of control neurons nearly doubled as compared with low-density cultures (Fig. $1 F, G)$. These results suggest that CLASP2 is a crucial regulator of axon growth and that CLASP2 might act through intrinsic mechanisms that control axon extension.

In contrast to the enhanced axon growth, dendrite development was impaired by knocking down CLASP2. Neurons depleted with CLASP2 developed fewer dendrites as compared with control neurons (Fig. 1C,E), and defects in dendrite development became more evident at a higher cell density (Fig. 1F,G). In contrast to control neurons that developed complex dendrites with increased arborization, dendrites in CLASP2-depleted neurons remained simple with few branches, suggesting a role for CLASP2 in dendritic development (Fig. 1F; EM Hur and FQ Zhou, unpubl.). Unlike CLASP2, knocking down CLASP1 had little effect on the length of axons and the number of dendrites (Fig. 1C-E), although both isoforms were expressed in cortical neurons (data not shown). Importantly, both the enhanced axon growth (see Fig. 5E, below) and the impaired dendrite development (Supplemental Fig. 1; EM Hur and FQ Zhou, unpubl.) induced by CLASP2 depletion were reversed by adding back the RNAi-resistant form of CLASP2. Together, these results identify CLASP2 as a pivotal regulator of neuronal morphogenesis that controls axon growth and dendrite development. 
Hur et al.

A

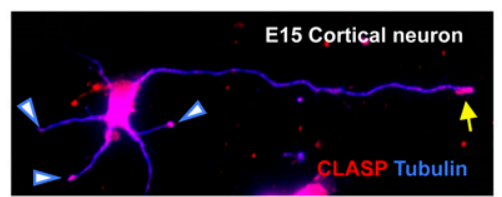

C
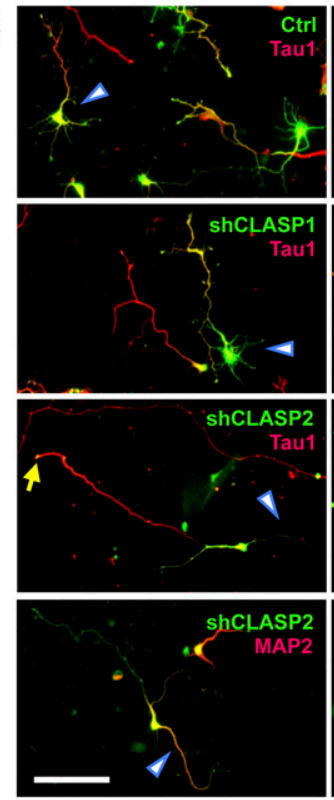

$\mathbf{F}$
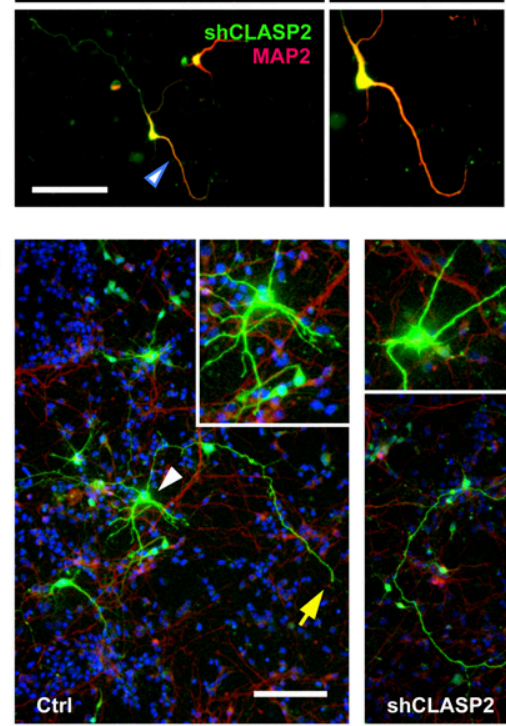

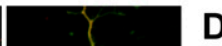

D

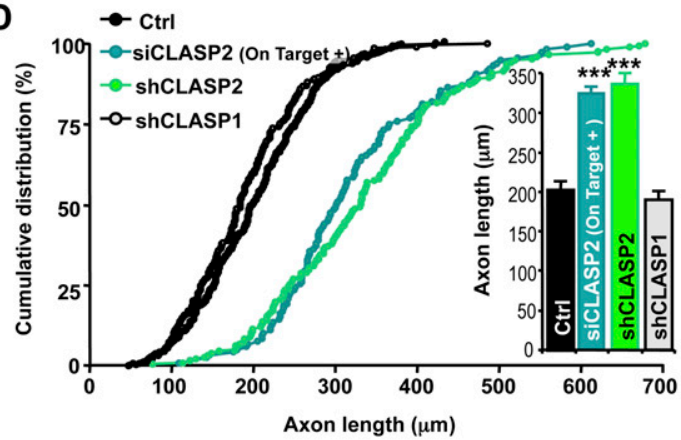

$\mathbf{E}$
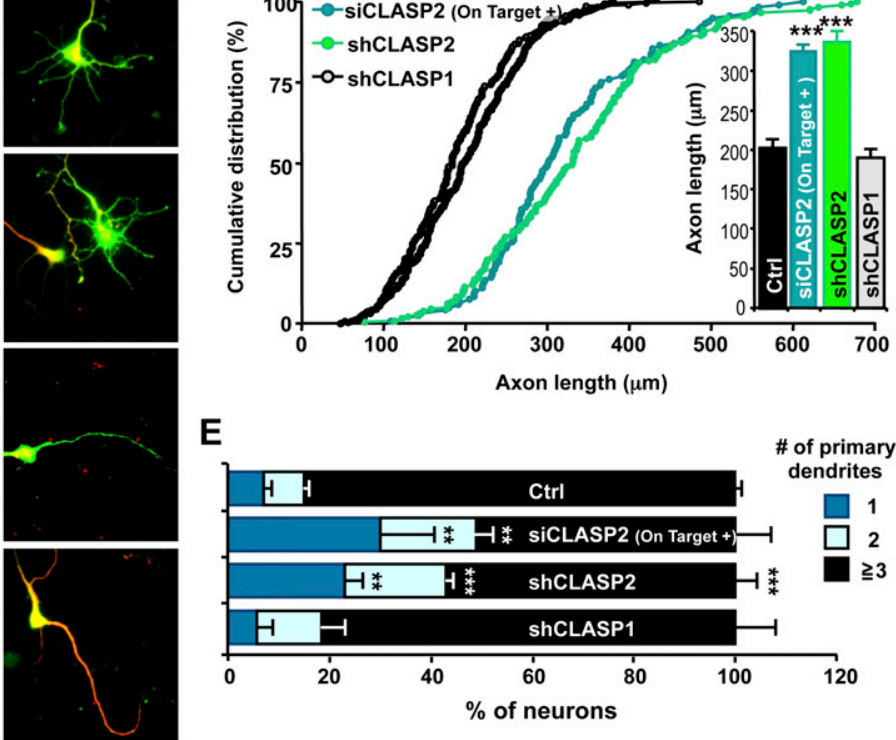

EGFP-CLASP2
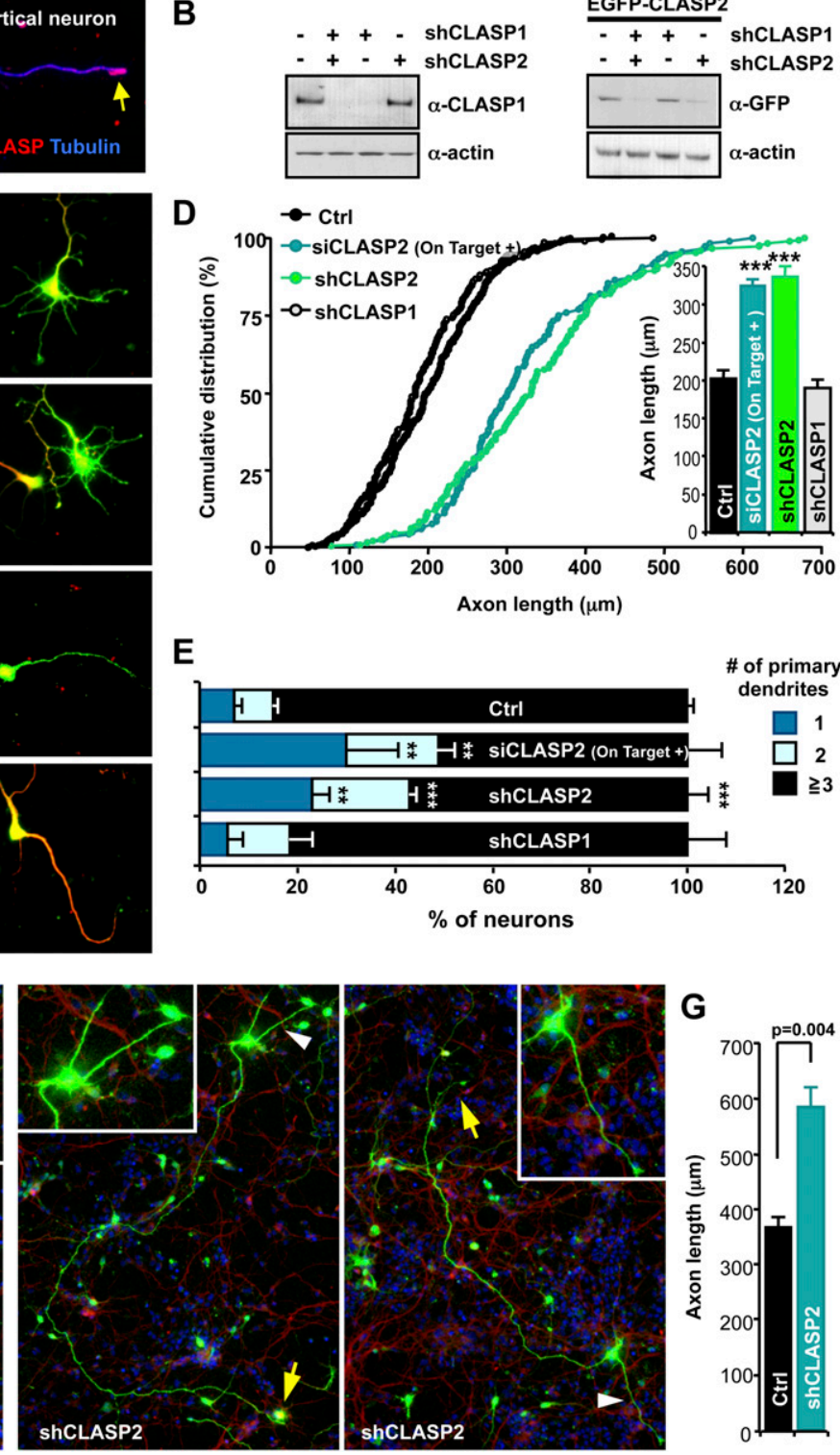

Figure 1. CLASP2 specifies neuronal morphogenesis by restricting axon extension and promoting dendritic arborization. $(A)$ Distribution of CLASP in embryonic day 15 (E15) cortical neurons. A representative immunostaining showing the enrichment of CLASP at the distal tips of an axon (arrow) and dendrites (arrowheads). (B) Validation of CLASP-shRNAs. CAD cells transfected with shRNAs alone (left) or together with EGFP-CLASP2 (right), as indicated, were subjected to Western blot analysis at $3 \mathrm{~d}$ after transfection. Representative blots are shown. $(C, D)$ E15 cortical neurons were transfected with shCLASP1, shCLASP2, siCLASP2 or control vector, as indicated, and fixed at $4 \mathrm{~d}$ in vitro (DIV4). Neurons were immunostained for an axonal marker, Tau1, or a dendritic marker, MAP2. Representative images $(C)$ and quantification of axon length $(D)$ are shown. $(C)$ Indicated areas (arrowheads) are enlarged (at right) to show clearly the dendrite morphology. Bar, $100 \mu \mathrm{m} .\left(^{\star \star \star}\right) P<0.001$ compared with control. $(E)$ Quantification of dendrite numbers of E15 cortical neurons expressing shCLASP1, shCLASP2, siCLASP2, or control vector. $\left(^{\star \star}\right) P<0.01$; $\left.\left.\right|^{\star \star \star}\right) P<0.001$ compared with control. $(F, G)$ E15 cortical neurons transfected with control vector or shCLASP2 were mixed with untransfected neurons for highdensity culture. Transfected neurons are shown in green. Cells were fixed and stained with anti-Taul antibodies (red) and Hoechst 33258 (blue). Note the markedly enhanced axonal growth (arrows) and impaired dendritic arborization (arrowheads, enlarged in insets) in CLASP2-depleted neurons. Representative images $(F)$ and quantification of axon length $(G)$ are shown. Bar, $100 \mu \mathrm{m}$.

GSK3 knockdown inhibits axon growth and induces MT reorganization via CLASP2

Several studies have reported that strong suppression of GSK3 activity impedes axon growth (Owen and GordonWeeks 2003; Kim et al. 2006), but the substrate(s) of GSK3 and the underlying mechanisms are not fully understood. Although GSK3 is known to phosphorylate CLASP2 and control CLASP-MT association (Wittmann and WatermanStorer 2005; Watanabe et al. 2009), the functional consequences of such regulation are unclear. Given the role of CLASP in inhibiting axon growth (see Fig. 1) and its 
connection with GSK3, we tested the possibility that CLASP2 might be responsible for the axon growth defect caused by GSK3 depletion. Consistent with a previous study (Kim et al. 2006), axon growth was drastically prevented by shGSK3 that down-regulated all of the GSK3 isoforms expressed in cortical neurons (Fig. 2A,B). Surprisingly, knocking down CLASP2 completely reversed the axon growth defect induced by GSK3 depletion (Fig. 2B,C), suggesting CLASP2 as a major substrate of GSK3 that mediates axon growth inhibition. In contrast to the suppression of axon extension, axonal branching has been shown to be promoted by pharmacological inhibition of GSK3 activity (Kim et al. 2006). We also observed increased axonal branching in response to GSK3 depletion, but this phenotype appeared to be independent of CLASP2, as shGSK3 increased the number of branches to a similar extent in the presence and absence of CLASP2 (Supplemental Fig. 2). Together, these results suggest that molecules other than CLASP2 might mediate the branching phenotype induced by GSK3 depletion and that CLASP2 is specifically required for the regulation of axon extension downstream from GSK3.

By examining the growth of axons by time-lapse video microscopy, we found that the rate of axon extension was significantly accelerated by knocking down CLASP2 but

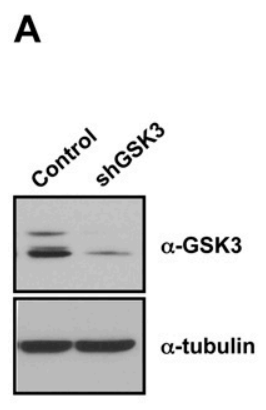

B

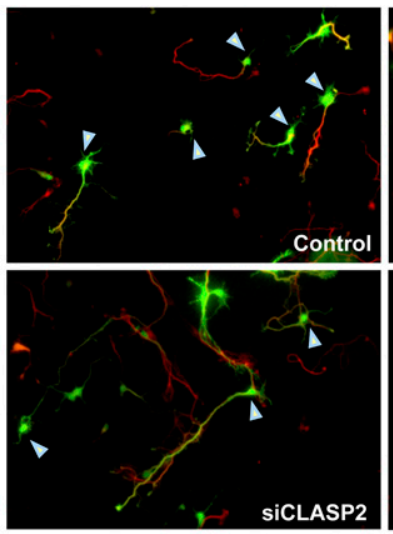

D
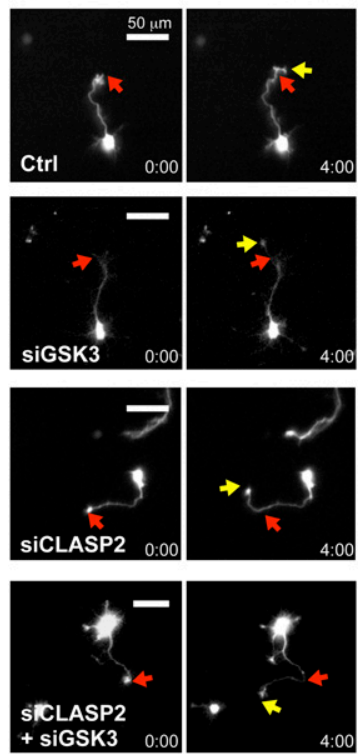
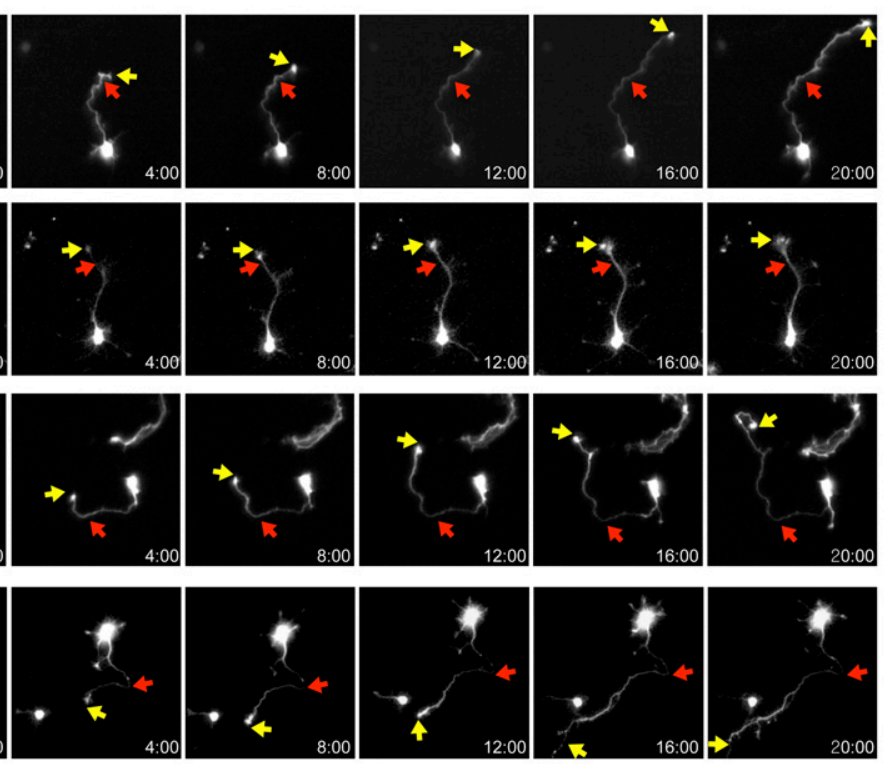
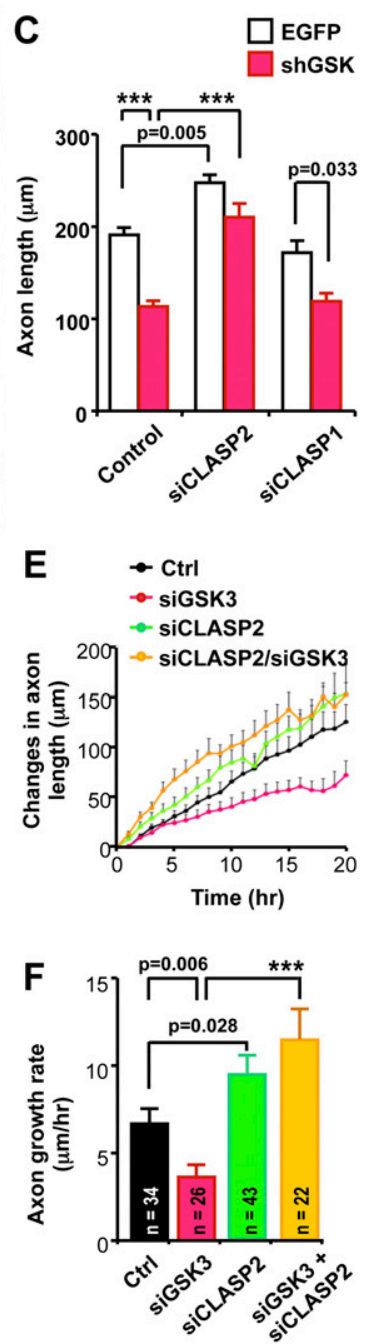

Figure 2. CLASP2 mediates axon growth inhibition induced by GSK3 knockdown. (A) Validation of shGSK3 in cortical neurons. E15 cortical neurons were transfected with shGSK3 or control vector and subjected to Western blot analysis at $4 \mathrm{~d}$ after transfection. Note that all shGSK3 isoforms (GSK3 $\alpha$, the highest band; GSK3 $\beta 1$ and GSK3 $\beta 2$, the lower two bands) expressed in cortical neurons are down-regulated by shGSK3. $(B, C)$ E15 cortical neurons were transfected with control vector, siCLASP1, siCLASP2, and/or shGSK3, as indicated, and fixed at DIV4. Transfected neurons are shown in green. Neurons were immunostained for an axonal marker, Tau1 (red). Representative images $(B)$ and quantification of axon length $(C)$ are shown. $\left.{ }^{\star \star \star}\right) P<0.001$. $(D-F)$ Time-lapse imaging of E15 cortical neurons transfected with control vector, siCLASP2, and/or siGSK3, as indicated, was performed as described in the Supplemental Material. Time-lapse image sequences of representative neurons are presented in $D$. Time is denoted in the bottom right corner. Red arrows indicate the position of growth cones when time lapse was initiated, and yellow arrows indicate growth cone positions at the indicated time points. Changes in axon length as a function of time $(E)$ and quantification of axon growth rate calculated from timelapse images $(F)$ are shown. 
was decelerated by depleting GSK3, relative to control neurons (Fig. 2D-F; Supplemental Movies 1-3). Importantly, the slower axon growth rate of GSK3-depleted neurons was completely reversed by knocking down CLASP2 (Fig. 2D-F; Supplemental Movie 4), demonstrating that CLASP2 regulates axon growth and mediates the axon growth inhibition induced by GSK3 depletion via controlling the rate of axon extension.

Axon growth rate varies as the axon extends, displaying periods of fast axon growth, slow growth or pausing, and retraction. Analyzing the changes in axon length from time-lapse images revealed that as compared with control neurons, knocking down GSK3 significantly increased the occurrences of slow-growing or pausing events (as defined by net axon length change of $<5 \mu \mathrm{m} / \mathrm{h}$ ) while decreasing the frequency of actively growing events (Supplemental Fig. 3). Both of these trends induced by GSK3 knockdown were completely reversed when CLASP2 was also depleted (Supplemental Fig. 3).

Pausing axons often form MT loops in the growth cones (Conde and Caceres 2009). Knocking down GSK3 doubled the percentage of growth cones containing looped MTs as compared with control neurons (Fig. 3A,B). In control growth cones where MTs formed loops $(22 \% \pm 5 \%$ of growth cones), MT loops were found in the central domain, whereas other MTs still protruded into the growth cone periphery. However, in GSK3-depleted growth cones, the formation of MT loops was much more prominent, with little MTs invading into the growth cone periphery. Notably, MTs were unable to form loops when CLASP2 was depleted, suggesting that the formation of MT loops requires CLASP2 and that CLASP2 plays an instructive role to control MT configuration in the growth cone. Moreover, knocking down CLASP2 prevented the prominent formation of MT loops induced by GSK3 depletion (Fig. 3A,B), suggesting that CLASP2 conveys the axon
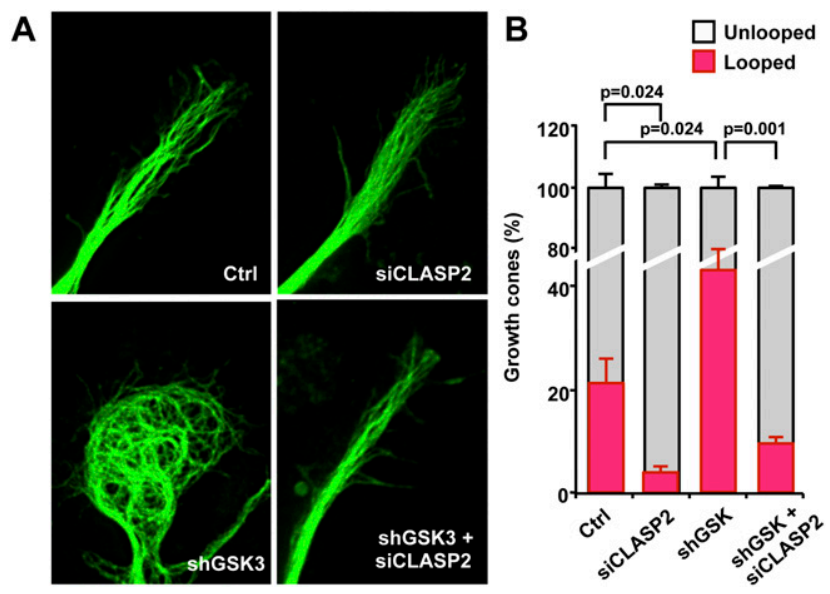

Figure 3. GSK3 knockdown induces MT looping via CLASP2. $(A, B)$ E15 cortical neurons transfected with control vector, siCLASP2, and/or shGSK3, as indicated, were immunostained for tubulin. Representative images $(A)$ and quantification of the percentage of growth cones showing MT looping $(B)$ are presented. growth inhibitory signal of shGSK3 by regulating MT structures in the growth cone.

\section{CLASP displays two distinct MT-binding activities in nerve growth cones}

Unlike many other +TIPs, endogenous CLASP binds along MT lattices in addition to tracking the plus ends (Wittmann and Waterman-Storer 2005). Through biochemical, molecular, and cell biological approaches, several studies have established that the lattice-binding and the plus endtracking activities reside in different domains of CLASP2 and that GSK3 is a key regulator of such MT-binding activities (Wittmann and Waterman-Storer 2005; Kumar et al. 2009; Watanabe et al. 2009). To examine MT-binding properties of CLASP in nerve growth cones, neurons were immunostained with anti-pan-CLASP antibodies that recognize both CLASP1 and CLASP2. Because it was difficult to distinguish individual MTs near the growth cone central domain, antibodies against EB1 were also applied to clearly mark the plus ends of MTs. From immunostaining, it was evident that endogenous CLASP displayed two distinct MT-binding activities. On some MTs, CLASP specifically marked the very tips of MT plus ends, displaying comet-like binding activities similar to EB1. Although both CLASP and EB1 labeled MT plus ends, these proteins did not colocalize: CLASP was always found proximal to EB1, often excluded from the region of highest EB1 intensity (Fig. 4A). On other MTs, CLASP-MT association extended beyond the plus ends, showing increased binding along the distal region of MT lattices (Fig. 4A). For the purpose of this study, we define this extended MT-binding activity of CLASP as "distal lattice binding," as opposed to "plus end binding," which is characterized by the sharply decaying comet-like MT-binding activity at the tips of the plus ends. Importantly, on the MTs where CLASP displayed distal lattice binding, a single EB1 signal was found at the tip and EB1 showed a clear plus end-binding activity, indicating that the extended binding of CLASP along the lattices was specific; i.e., not resulting from poor resolution of individual MT plus ends, which would otherwise be labeled with multiple EB1 signals.

Plotting the fluorescence intensity of CLASP as a function of increasing distance from the MT end confirmed the two distinct MT-binding profiles (Fig. 4B). CLASP decorating the very tips of the plus ends showed intensity decay profiles analogous to EB1, displaying sharply decreasing fluorescence intensity toward the baseline after an initial peak in fluorescence (Fig. 4B, top panels). In contrast, the absolute amount of fluorescence of CLASP that displayed distal lattice binding was usually maintained along MTs at a similar level for $\sim 2 \mu \mathrm{m}$ (Fig. 4B, bottom panels), and average length of the distal lattice bindings was significantly longer than that of the plus end bindings (Fig. 4C). Furthermore, the distribution of CLASP lengths bound to MTs resulted in a doublepeaked histogram (Fig. 4D), reflecting the two distinct populations. In contrast, EB1 always displayed comet-like binding activity at MT plus ends throughout the growth cone (Fig. 4A-C), and distribution of EB1 lengths resulted in a single-peaked histogram (Fig. 4E). 
A

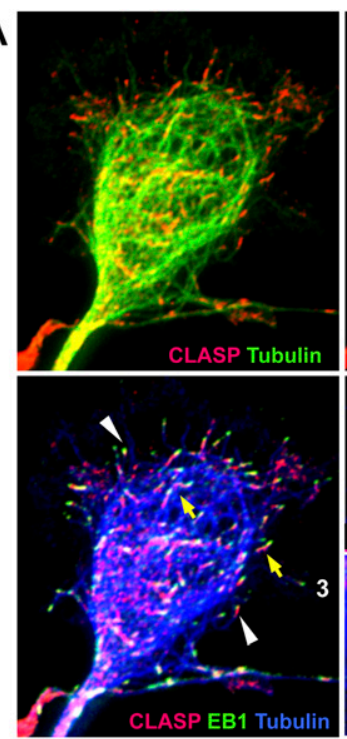

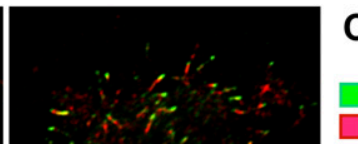

Distal lattice-binding
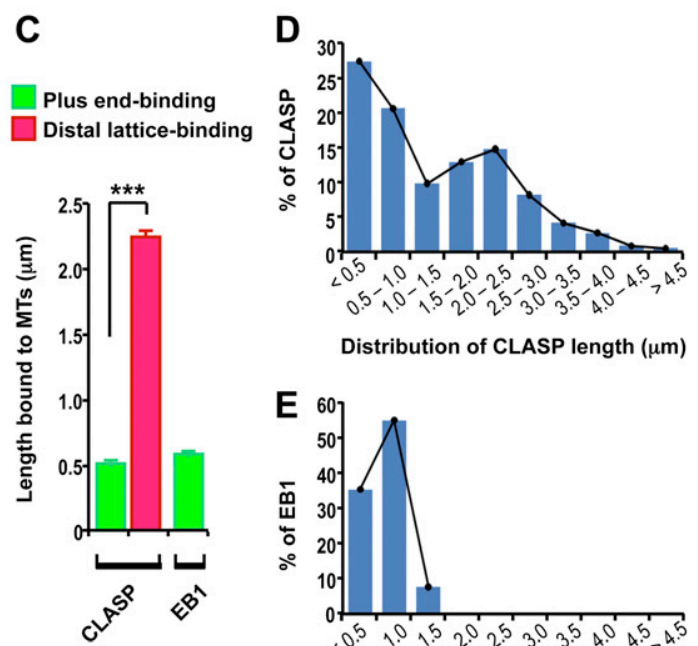

Distribution of CLASP length $(\mu \mathrm{m})$

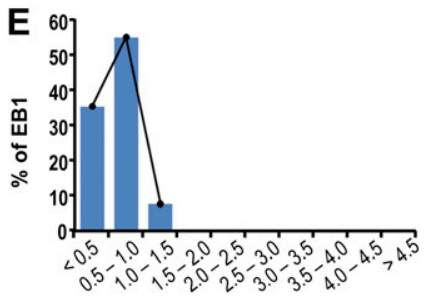

Distribution of EB1 length $(\mu \mathrm{m})$
B

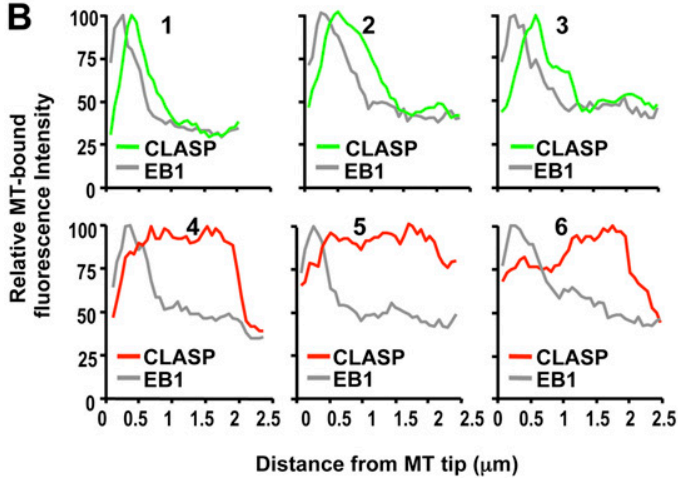

$\mathbf{F}$

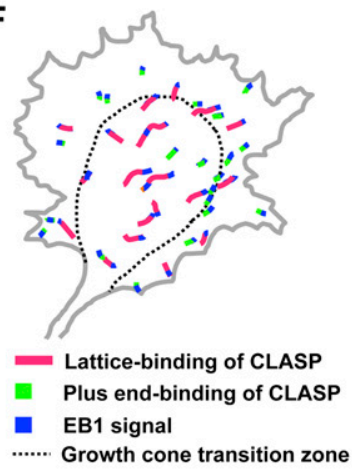

$\mathbf{G}$
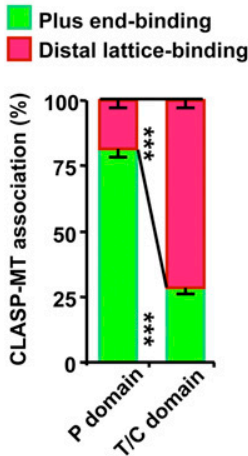

Figure 4. CLASP displays two distinct MT-binding activities in growth cones of embryonic cortical neurons. (A) Representative images of an E15 cortical growth cone immunostained for CLASP, EB1, and tubulin. Plus end-binding (white arrowheads) and latticebinding (yellow arrows) activities are enlarged in the bottom right panels. Bar, $1 \mu \mathrm{m}$. $(B)$ Fluorescence intensity profiles of CLASP and EB1. CLASP and EB1 signals numbered in $A$ were quantified by intensity line scans to present the relative fluorescence intensity profiles. $(C)$ Average length of endogenous CLASP and EB1 bound to MTs in growth cones. Signals were categorized as "plus end binding" or "distal lattice binding" on the basis of the fluorescence intensity profiles as described in the Results section. $(D, E)$ Histogram distribution of lengths of CLASP $(D)$ and EB1 $(E)$ bound to MTs. $(F, G)$ Spatial distribution of plus end and distal lattice bindings of endogenous CLASP in growth cones of cortical neurons. The gray line shows the growth cone's boundary, and dashed lines indicate the transitional zone. CLASP-MT association in a representative growth cone $(F)$ and quantification of MT-binding activities in multiple growth cones $(G)$ are shown. $\left(^{\star \star \star}\right) P<0.001, \chi^{2}$ test.

The lattice-binding activity of CLASP2 mediates MT looping and axon growth inhibition

Although the spatial distribution of the plus end binding and the lattice binding was not exclusive, the majority of CLASP proteins in the growth cone periphery specifically decorated MT plus ends, whereas those near the growth cone center preferred distal lattice binding (Fig. 4F,G). When full-length CLASP2 (EGFP-CLASP2-FL) was expressed in a neuronal cell line, CAD, MTs failed to extend into the cell periphery and formed loops when CLASP bound along the lattices, but not when CLASP's binding was restricted to MT plus ends (Supplemental Fig. 4A). In contrast to the changes in MT configuration induced by CLASP, overexpression of two other +TIPs, EB1 and APC, had little effect (Supplemental Fig. 4B,C).
The association between the lattice-binding activity of CLASP and MT loop formation (Supplemental Fig. 4A), the spatial segregation of the lattice-binding and the plus end-binding activity of CLASP (Fig. 4G), and the instructive role of CLASP in controlling MT configuration in the growth cone (Fig. 3) led us to hypothesize that the latticebinding activity of CLASP might function to restrain MTs from penetrating into the growth cone periphery. To test this, we first examined the effects of several CLASP2 mutants (Fig. 5A) on growth cone MT structures. MT plus end-binding activity of CLASP2 is mediated by the plus end-binding $(\mathrm{M})$ domain and/or the $\mathrm{C}$-terminal $(\mathrm{C})$ domain, whereas the lattice-binding activity requires both the $\mathrm{M}$ and the lattice-binding (L) domains (Mimori-Kiyosue et al. 2005; Wittmann and Waterman-Storer 2005). Because ectopic expression of some of the CLASP2 mutants affected 
Hur et al.

A

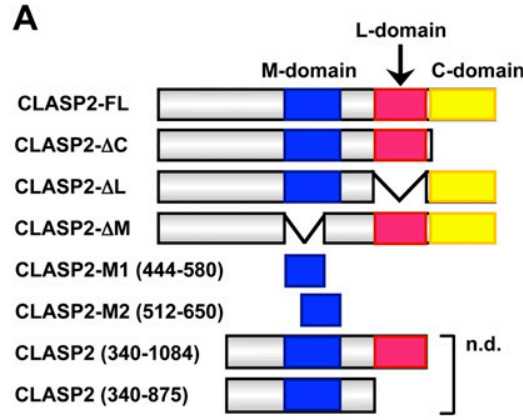

C

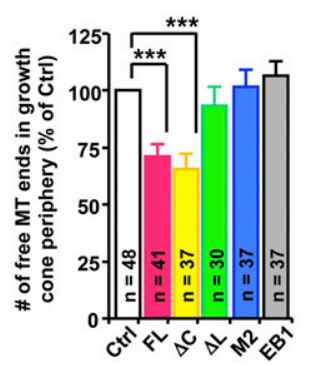

D

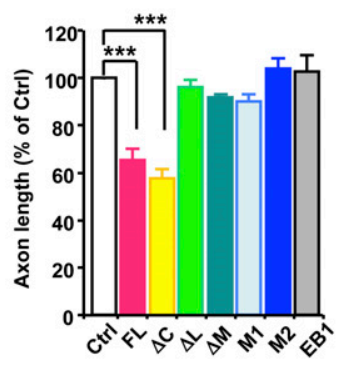

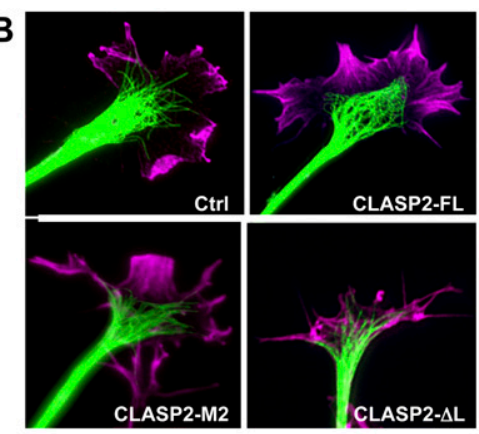

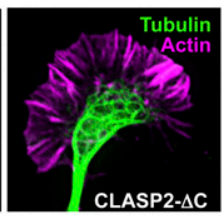

E

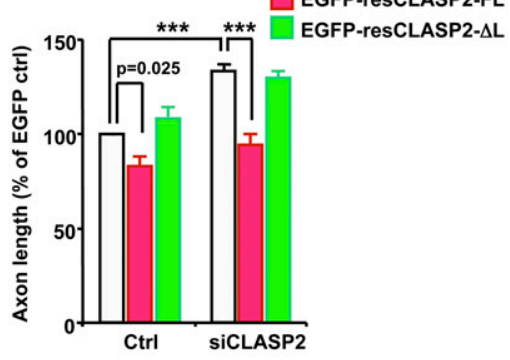

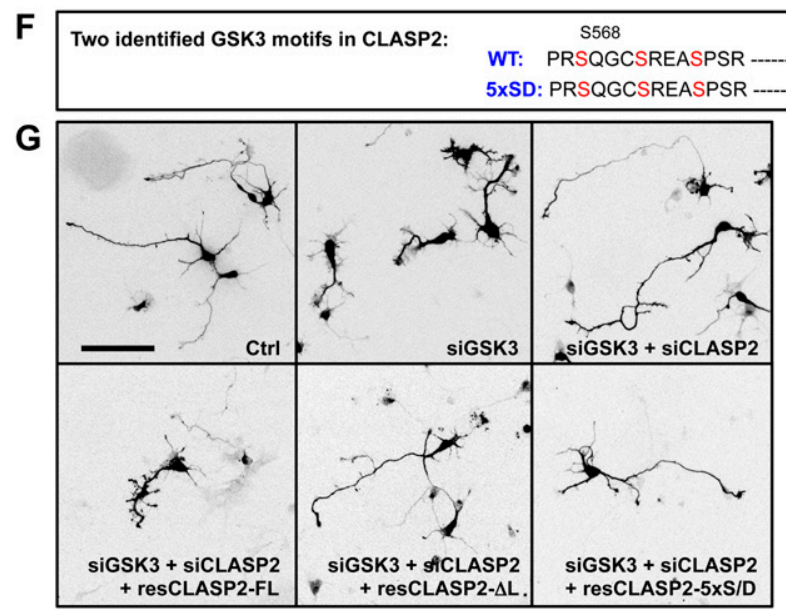

S594

$\mathrm{S} 610$

STCSEASRESSRDTSPV

VDQGCDREADRESDRDTDPV

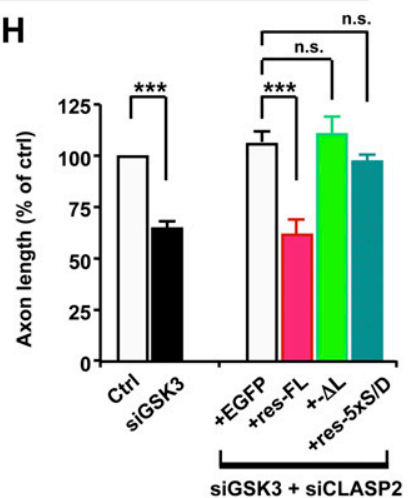

Figure 5. MT lattice-binding activity of CLASP2 prevents MT protrusion toward growth cone periphery and inhibits axon growth. $(A)$ Schematic of CLASP2 constructs used in this study. Effects of two constructs denoted "n.d." could not be determined because of protein aggregation and cell death after transfection. (B) Representative images of E15 DRG growth cones overexpressing the indicated constructs. (C) Quantification of MT structures in growth cones. The number of free MT ends in the growth cone P domain expressing the indicated constructs was quantified and normalized against the size of the growth cone as described in the Materials and Methods. The numbers of growth cones analyzed are shown. (D) Effects of CLASP2, its deletion constructs, and EB1 on axon growth. E15 DRG neurons were transfected as indicated and were cultured overnight. Axon length is presented as a percentage of EGFP-transfected control. (E) E15 cortical neurons were transfected with control or siCLASP2 together with EGFP, EGFP-resCLASP2-FL, or EGFP-resCLASP2- $\Delta$ L, as indicated, and fixed at DIV4. siRNA oligos targeting the L domain of CLASP2 (Mimori-Kiyosue et al. 2005) was used to deplete endogenous CLASP2. Axon length was measured and is presented as a percentage of EGFP-transfected control. $(F)$ Amino acid sequences of the two identified GSK3 motifs in wild-type (WT) CLASP and resCLASP-5xS/D mutant (GSK3 sites in red). The numbering of amino acid corresponds to previous literature (Kumar et al. 2009). Mutation sites are underlined. $(G, H)$ E15 cortical neurons were transfected with control, siGSK3, and/or siCLASP2, together with variant mutants of CLASP2 that are resistant to siCLASP2, as indicated, and fixed at DIV4. Representative images $(G)$ and quantification of axon length $(H)$ are presented. Bar, $100 \mu \mathrm{m}$. $\left(^{\star \star \star}\right) P<0.001$; (n.s.) statistically not significant.

neuronal polarization in cortical neurons, we used embryonic DRG neurons to avoid a complicated interpretation regarding polarity. In control DRG neurons, tightly bundled axonal MTs typically splayed as they entered the growth cone, and a subset of MTs extended into the growth cone periphery. However, ectopic expression of EGFP-
CLASP2-FL or EGFP-CLASP2- $\Delta$ C, the constructs that display lattice-binding (Wittmann and Waterman-Storer 2005), prevented MT extension into the growth cone periphery, often causing MT looping (Fig. 5B,C). In contrast, EGFPCLASP2- $\Delta \mathrm{L}$, which retains only the plus end-tracking but not the lattice-binding activity (Supplemental Fig. 4D), 
failed to induce any changes in the configuration of growth cone MTs. In addition, MT structures were largely unaffected by EGFP-CLASP2-M2 or EGFP-CLASP2- $\Delta$ M, the constructs that do not bind along MT lattices (Fig. 5C; Supplemental Fig. 5). Similar to MT protrusion, axon growth was inhibited only by EGFP-CLASP2-FL and EGFPCLASP2- $\Delta$ C, but not by any other mutants that lacked the lattice-binding activity (Fig. 5D). To test whether the lattice-binding activity of CLASP is sufficient to induce the changes in growth cone MT configuration and inhibit axon growth, we transfected the neurons with the shortest fragment of CLASP2 (containing amino acids 340-1084) that has been tested to bind to MT lattices (Wittmann and Waterman-Storer 2005). This fragment of CLASP2, however, induced the formation of aggregates and caused cell death in neurons, precluding further analysis of the role of this fragment in controlling MT configuration and axon growth. In contrast to CLASP2, neither growth cone MT structures nor axon growth was affected by EGFP-EB1. None of the constructs we tested had any noticeable effects on actin-based structures, such as filopodia and lamellipodia. Together, these results suggest that the lattice-binding activity of CLASP2 induces the reorganization of growth cone MTs and inhibits axon growth.

GSK3 inhibition impedes axon growth through the lattice-binding activity of CLASP2

As in DRG neurons, axon growth in cortical neurons was inhibited by CLASP2-FL but not CLASP2- $\Delta$ L (Fig. 5E). Moreover, by performing rescue experiments with siRNAresistant constructs of CLASP2, we found that the enhanced axon growth induced by knocking down CLASP2 was completely reversed by adding back resCLASP2-FL, but not resCLASP2- $\Delta \mathrm{L}$, providing further support for the requirement of the lattice-binding activity to suppress axon growth. Similarly, depletion of GSK3 restricted axon growth only in the presence of resCLASP2-FL, but not resCLASP2- $\Delta \mathrm{L}$, when these constructs were expressed in neurons depleted with both CLASP2 and GSK3 (Fig. 5G,H). In addition, CLASP's binding along MT lattices was greatly increased in GSK3-depleted growth cones (Supplemental Fig. 6). Collectively, these results indicate that the axon growth defect induced by GSK3 depletion is mediated by the latticebinding activity of CLASP2.

Many substrates of GSK3 harbor multiple consecutive GSK3 sites (S/T-X-X-X-S/T) that are sequentially phosphorylated by GSK3. Extensive biochemical approaches have confirmed that two clusters of such sites in CLASP2 are phosphorylated by GSK3 (Fig. 5F) and that complete phosphorylation of all GSK3 sites disrupts both the plus end-tracking and the lattice-binding activities of CLASP2 (Kumar et al. 2009). However, phosphorylation of serine residues in only one of the two GSK3 motifs specifically abrogates the lattice-binding activity, while retaining the plus end-binding activity (Kumar et al. 2009). We also confirmed that introducing phosphomimetic mutations into only one of the two GSK3 motifs in resCLASP2-FL (resCLASP2-5xS/D) (Fig. 5F) specifically prevented lattice-binding activity while preserving the plus end-track- ing activity (Supplemental Fig. 4E). Consistent with the lack of lattice-binding activity, resCLASP2-5xS/D did not inhibit growth cone MT protrusion into the growth cone periphery (Supplemental Fig. 5) and failed to reverse the axon growth promotion induced by siCLASP2 (Supplemental Fig. 7). Importantly, when the endogenous CLASP2 was replaced with the resCLASP2-5xS/D mutant, siGSK3 was unable to inhibit axon growth (Fig. 5F,H), providing ample evidence that the attenuation of axon growth induced by GSK3 depletion requires the lattice-binding activity of CLASP2.

Impaired axon growth induced by GSK3 inhibition or CLASP2 overexpression is completely reversed by blocking myosin II activity

The results presented so far suggest that the increased lattice-binding activity of CLASP, induced by either CLASP overexpression or GSK3 inhibition, impedes axon growth by preventing MT protrusion into the growth cone periphery. If the blockade of $\mathrm{MT}$ protrusion is responsible for inhibiting axon growth, then forcing MT extension into the growth cone periphery would be able to rescue the axon growth defect under such conditions. The actin cytoskeleton is an important factor that controls MT configuration in the growth cone, and particularly, retrograde flow of actin powered by myosin II carries MTs rearward, thus acting as a dynamic barrier to MT extension (Schaefer et al. 2002; Burnette et al. 2007; Hur et al. 2011). When neurons were treated with a specific inhibitor of myosin II, blebbistatin (Straight et al. 2003; Allingham et al. 2005), MT invasion into the growth cone periphery was dramatically increased, and importantly, axon growth was no longer inhibited by CLASP2 overexpression or GSK3 inhibition (Supplemental Fig. 8). These results suggest that reorganization of growth cone MTs-i.e., blockade of MT extension into the periphery-induced by either manipulation underlies axon growth inhibition.

The plus end-binding activity of CLASP1/2 supports axon growth by stabilizing MTs

Contrasting with the expected role of CLASP as a +TIP, here we suggest that CLASP2 inhibits axon growth and that this unexpected role of CLASP requires its unique lattice-binding activity. What role would CLASP have in the regulation of axon growth if it binds to MT plus ends? Actually, when endogenous CLASP2 was replaced by CLASP2- $\Delta$ L or CLASP-5xS/D, mutants that retain the plus end-binding activity but not the lattice-binding activity, axon growth was enhanced compared with control (Fig. 5E; Supplemental Fig. 7), suggesting that the plus endbinding activity might have an axon growth-promoting function. It was, however, difficult to unequivocally address the role of the plus end-binding activity of CLASP2 in developing cortical neurons because the plus end-binding activity was confounded by the prominent lattice-binding activity. Fortunately, we found that in the growth cones of adult DRG neurons, endogenous CLASP almost exclusively ( $>90 \%$ of growth cones) displayed plus end-binding activity (Fig. 6A-C). Given that GSK3 activity and the 
Hur et al.
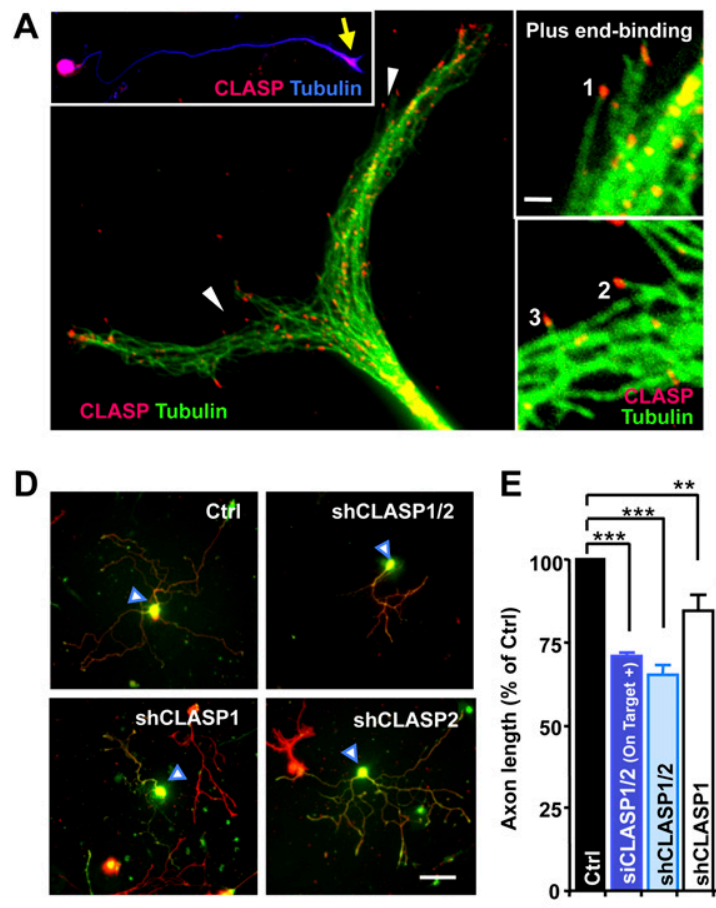

$\mathbf{G}$

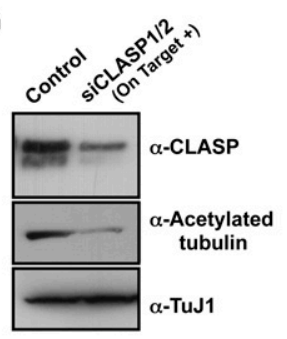

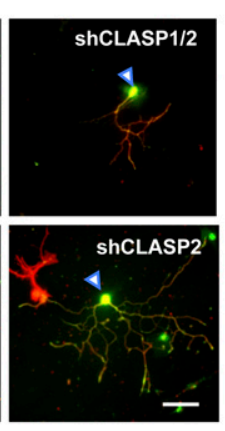

E

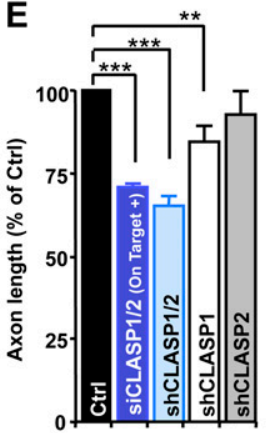

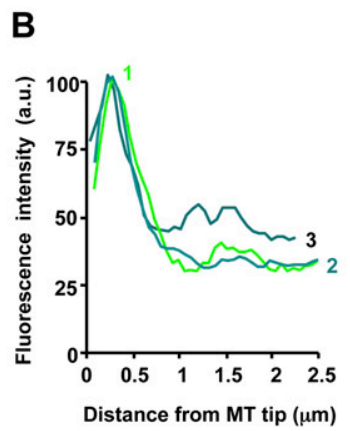
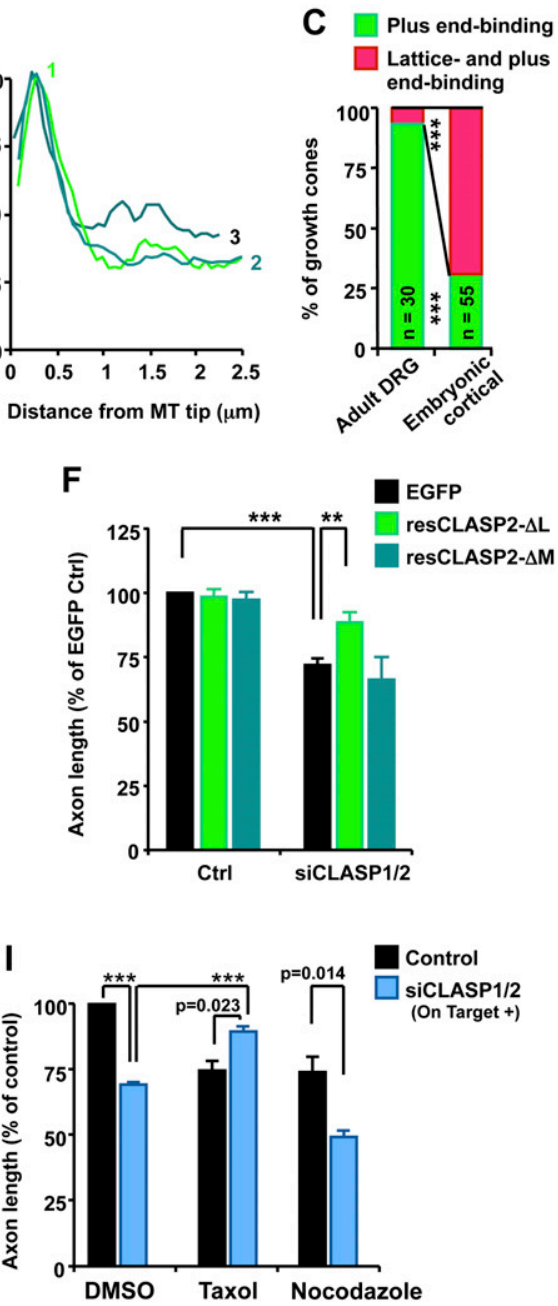

Figure 6. MT plus end-binding activity of CLASP1/2 supports regenerative axon growth. (A) Representative images of CLASP immunostaining in adult DRG neurons showing its enrichment in the growth cone (inset, arrow). MT plus end bindings (arrowheads) are enlarged in the top and bottom right panels. Bar in the inset, $1 \mu \mathrm{m}$. (B) Fluorescence intensity profiles of CLASP. CLASP signals numbered in $A$ were quantified by intensity line scans to present the relative fluorescence intensity profiles. $(C)$ Quantification of plus end-binding and lattice-binding activities of CLASP in nerve growth cones. Embryonic cortical and adult DRG neurons were immunostained side by side for CLASP and tubulin, and CLASP-MT association was quantified as described in the Materials and Methods. Numbers of growth cones analyzed are shown. $\left(^{\star \star \star}\right) P<0.001, \chi^{2}$ test. $(D, E)$ Adult DRG neurons were transfected as indicated and replated at DIV4 to allow axon growth anew. Cells were fixed at 18-20 h after replating and stained for neuronal tubulin, TuJ1 (red). Transfected neurons (arrowheads) are shown in green. Representative images $(D)$ and quantification of axon length $(E)$ are shown. Bar, 100 $\mu \mathrm{m}$. (F) Adult DRG neurons were transfected as indicated and replated at DIV4 to reinitiate axon growth. To deplete endogenous CLASP1 and CLASP2, the ON-TARGETplus siRNA pool against CLASP1 and siRNA oligos targeting the L domain of CLASP2 were used. Cells were fixed at $18-20 \mathrm{~h}$ after replating and axon length was measured. $\left(^{\star \star}\right) P<0.01 ;\left(^{\star \star \star}\right) P<0.001$. $(G, H)$ Adult DRG neurons were transfected with either EGFP alone (control) or together with siCLASP1 and siCLASP2 (siCLASP1/2, both ON-TARGETplus siRNA pools). Neurons were subjected to Western blot analysis at DIV4 $(G)$ or immunocytochemistry $(H)$ with the indicated antibodies. For $H$, fluorescence intensities of acetylated and tyrosinated tubulin in the growth cone were measured and presented as a ratio (MT stability index). (I) Adult DRG neurons transfected with either EGFP alone (control) or together with siCLASP1/2 (both ON-TARGETplus siRNA pools) were replated at DIV4 and allowed to grow axons anew. Taxol or nocodazole was treated at the time of replating. Neurons were fixed at $18-20 \mathrm{~h}$ after replating and axon length was measured. $\left(^{\star \star \star}\right) P<0.001$.

expression level of CLASP are important parameters that determine CLASP-MT association (Supplemental Fig. 9A,B), we compared the two factors in adult DRG and embryonic cortical neurons. Interestingly, Ser 9 phosphorylation of GSK3 3 , an inhibitory phosphorylation that inversely correlates with GSK3 activity, was significantly lower in adult DRG neurons as compared with embryonic cortical neurons, suggestive of higher GSK3 activity in adult neurons (Supplemental Fig. 9C,D). In addition, imunofluorescence microscopy revealed reduced labeling of CLASP in growth cones of adult DRG neurons relative to those of embryonic cortical neurons (Supplemental Fig. 9E). Given that both the higher GSK3 activity and the lower CLASP protein level in the growth cones of adult DRG 
neurons are the conditions that bias CLASP's binding toward MT plus end binding (Supplemental Fig. 9A,B), these two factors might contribute to the different MTbinding activities observed in embryonic cortical and adult sensory neurons.

The exclusive binding of CLASP to MT plus ends in adult DRG neurons allowed us to examine the possible role of the plus end-binding activity of CLASP in the regulation of axon growth. For this purpose, adult DRG neurons were transfected with siRNAs against CLASP1 and/ or CLASP2, cultured for $4 \mathrm{~d}$ to deplete endogenous CLASP, and then replated to assess axon growth anew. Control neurons regenerated axons robustly without the addition of any growth factors, whereas neurons depleted with both CLASP1 and CLASP2 showed substantially attenuated axon growth (Fig. 6D,E). Knocking down CLASP1 alone resulted in a less severe phenotype as compared with double knockdown, and depletion of CLASP2 alone did not cause a statistically significant inhibition, suggesting redundant roles of CLASP1 and CLASP2 in promoting regenerative axon growth of adult DRG neurons. Furthermore, resCLASP2- $\Delta \mathrm{L}$ (resistant to both siCLASP1 and siCLASP2) was able to rescue the impaired axon growth induced by CLASP1/2 double knockdown (Fig. 6F). In contrast, resCLASP2- $\Delta \mathrm{M}$, a construct that does not display strong MT plus end-binding activity (Mimori-Kiyosue et al. 2005), had no effect. Together, these results provide further support for the notion that the plus end-binding activity of CLASP functions to promote axon extension.

Because the main function of CLASP as a +TIP is to protect and stabilize the growing ends of MTs (Galjart 2005), we then examined whether the axon growth-promoting function of CLASP is attributed to its effect on MT stability. Western blot analysis showed that knocking down both CLASP1 and CLASP2 substantially reduced the level of acetylated tubulin (Fig. 6G). Immunofluorescence microscopy also revealed that in contrast to low levels of taxol, knocking down CLASP1/2 decreased the ratio of acetylated versus tyrosinated tubulin in distal axons (Fig. 6H), suggestive of decreased MT stability in CLASP1/2-depleted growth cones. Importantly, low levels of taxol rescued the attenuated axon growth induced by CLASP $1 / 2$ knockdown, even though treatment with taxol alone inhibited axon growth (Fig. 6I). Conversely, treatment with nocodazole inhibited axon growth to a greater extent in CLASP1/2-depleted neurons. Together, these results suggest that the plus end-binding activity of CLASP1/2 supports axon extension by stabilizing axonal MTs.

\section{CLASP1/2 is required for rapid axon regeneration in vivo}

To extend these findings in vivo and examine the possible role of CLASP during axon regeneration of adult sensory neurons, we developed a novel technique that allows acute regulation of gene expression in DRGs of adult mice by directly electroporating genes of interest (Fig. 7A). Employing this technique, shCLASP1 and shCLASP2 were injected and transfected into the DRGs and, $3 \mathrm{~d}$ later, the mice were subjected to a sciatic nerve crush procedure.
Because shRNA plasmids also encode Venus, transfected sensory axons were fluorescently labeled, thus allowing direct measurement of the lengths of regenerating axons. When axon regeneration was assessed at $5 \mathrm{~d}$ after the nerve crush, axon regeneration of sensory neurons was drastically impaired in the mice that had been transfected with shCLASP1/2 (Fig. 7B,C), demonstrating that CLASP1/2 are required for efficient axon regeneration in vivo.

\section{MT-binding activity and functionality of CLASP are altered by regulation of GSK3}

Here we suggest that the role of CLASP to either support or inhibit axon growth is established by its MT-binding activity, which depends on the level of GSK3 activity (Fig. 7D). To further validate the model, we examined whether we could alter CLASP functionality by manipulating GSK3 activity. First, in adult DRG neurons, CLASP promotes regenerative axon growth, a function that is attributed to the exclusive plus end-binding activity of CLASP. If a relatively high GSK3 activity in these neurons (see Supplemental Fig. 9C,D) contributes to the exclusive plus end binding of CLASP, the model predicts that we should be able to convert CLASP functionality from being supportive to restrictive in axon growth by lowering GSK3 activity. Indeed, inhibition of GSK3 increased the lattice-binding activity of CLASP in adult DRG neurons (data not shown) as in COS cells and in cortical neurons (Supplemental Figs. 6, 9B). Importantly, inhibition of GSK3 markedly impeded regenerative axon growth, and this axon growth defect was fully rescued by depleting CLASP2 (Supplemental Fig. 10A). These results suggest that when GSK3 activity is greatly suppressed, CLASP2 mediates axon growth inhibition in adult DRG neurons, as opposed to its normal axon growthpromoting function in these neurons.

Second, in cortical neurons, contrasting with the role of CLASP in adult sensory neurons, CLASP2 restricts axon growth by binding along MT lattices. Interestingly, we found that GSK3 $\beta$-R96A, a GSK3 mutant that is capable of phosphorylating the unprimed but not the primed substrates (Frame et al. 2001), specifically reduced the latticebinding activity but not the plus end-binding activity of CLASP2 (Supplemental Figure 9B). If the model is correct, the GSK3 $\beta$-R96A mutant should enhance axon growth in cortical neurons. As predicted by the model, the GSK3 $\beta$ R96A mutant promoted axon growth in cortical neurons but not in adult DRG neurons (Supplemental Fig. 10B,C), where CLASP binding was already restricted to MT plus ends (see Fig. 6A-C). GSK3 $\beta$-R96A, however, failed to further increase axon growth in cortical neurons when CLASP2 was depleted, suggesting that the axon growth promotion induced by GSK3 $\beta$-R96A requires CLASP2 and occurs by regulating CLASP-MT association. Together, these results provide further support for the model (Fig. 7D) in which CLASP functionality is determined by GSK3dependent regulation of the MT-binding activity of CLASP.

\section{Discussion}

The present study reveals that CLASP is a crucial regulator of neuronal morphogenesis and identifies CLASP2 
Hur et al.
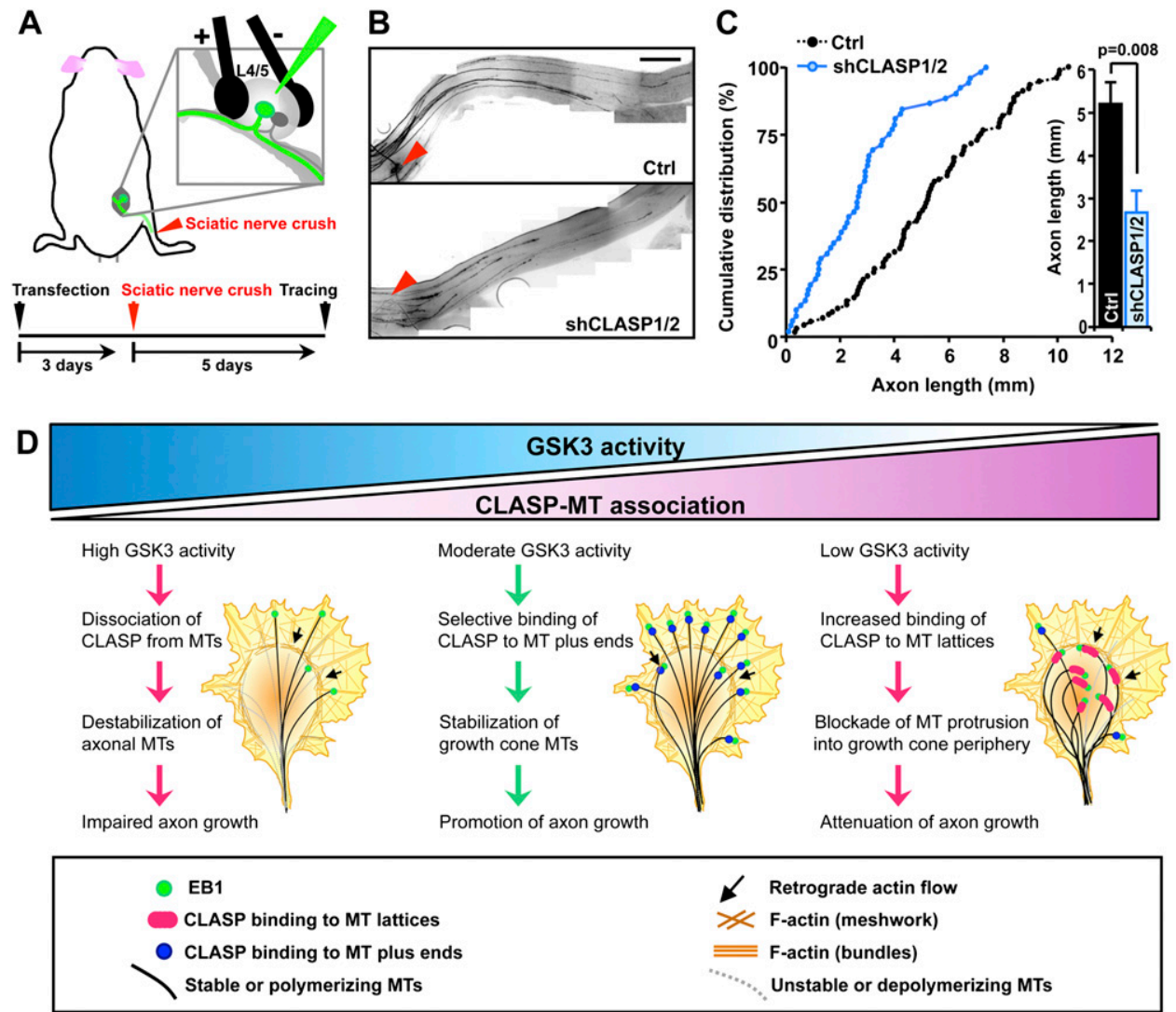

Figure 7. CLASP1/2 are required for rapid axon regeneration in vivo. (A) Schematics of the experimental protocol for the in vivo electroporation and investigation of axon regeneration. (B) After transfection of control vector or shCLASP1 and shCLASP2 (shCLASP1/2) into the DRGs, mice were subjected to a sciatic nerve crush procedure as depicted in $A$. Using the whole-mount nerve segment, the lengths of all identifiable regenerating axons transfected with either control or shCLASP1/2 (encoding Venus) were measured from the crush site (marked by epineural suture, arrowheads) to the distal axon tips. Representative images of sciatic nerves $(B)$ and quantification of axon regeneration length (five mice for each condition) $(C)$ are shown. Bar, $1 \mathrm{~mm}$. (D) A working model for GSK3-CLASP-MT triad in the regulation of axon growth. A detailed description of the model is discussed in the text.

as a physiological target of GSK3 to exert control over axon growth. We also showed that CLASP differentially controls developmental and regenerative axon growth, depending on its MT-binding activity. Furthermore, through a comprehensive approach encompassing molecular and cell biology, we tackled the underlying mechanisms involved and propose a model in which CLASP acts as a molecular sensor that detects different levels of GSK3 activity to control axon extension by regulating the stability and organization of growth cone MTs.

\section{GSK3 depletion inhibits axon growth by regulating the lattice-binding activity of CLASP2}

In cortical neurons, knocking down CLASP2 markedly accelerated axon growth and completely reversed the attenuated growth induced by GSK3 depletion. Several lines of evidence suggest that CLASP restricts axon extension via its lattice-binding activity. Gain-of-function studies show that MT protrusion into the growth cone periphery and the subsequent axon growth were inhibited only by CLASP2 constructs that are capable of binding along the lattices. Axon growth was inhibited by resCLASP2-FL but not resCLASP2- $\Delta \mathrm{L}$ when these constructs were expressed in CLASP2-depleted neurons. Similarly, when endogenous CLASP2 was replaced by different CLASP2 constructs in cortical neurons, siGSK3 prevented axon growth only in the presence of resCLASP2-FL, but not resCLASP2- $\Delta$ L or resCLASP2-5xS/D-mutants that do not associate with MT lattices. In fact, resCLASP2- $\Delta$ L or resCLASP2-5xS/D fully rescued the axon growth defect caused by GSK3 depletion, suggesting that the latticebinding activity of CLASP2 is required for the axon growth inhibition.

Suppression of axon growth induced by the lattice-binding properties of CLASP might be due to overstabilization of MTs or the structural changes of MTs in the growth cone or a combination of the two. Given that neither CLASP2 overexpression nor GSK3 inhibition impeded axon growth in the presence of blebbistatin, which is also known to stabilize MTs (Even-Ram et al. 2007), we favor the hypothesis that the structural reorganization of growth cone MTs underlies axon growth inhibition. However, further studies will be needed to clearly distinguish the two possibilities.

The configuration of growth cone MTs is modulated by the actin cytoskeleton, and increasing evidence suggests 
that intricate molecular interactions exist between the two cytoskeletal networks during neuronal morphogenesis (Arimura and Kaibuchi 2007; Geraldo et al. 2008; Conde and Caceres 2009; Stiess and Bradke 2011). Recent studies have suggested plausible links between CLASP and actin-binding proteins, such as zipper (Drosophila ortholog of myosin II), IQGAP1, and ACF7 (Drabek et al. 2006; Watanabe et al. 2009; Lowery et al. 2010). CLASP has also been shown to bind directly to actin filaments and undergo retrograde flow in Xenopus growth cones (Tsvetkov et al. 2007). Interestingly, our results suggest that myosin II activity is required for CLASP2 to induce the structural reorganization of growth cone MTs and inhibit axon growth, implying an interaction of CLASP2 with myosin II-mediated actin reorganization and/or retrograde actin flow. The exact molecular mechanisms, however, that underlie the coupling of CLASP2 to actin and the relevance in mediating MT looping and subsequent axon growth inhibition require further investigation.

Previous literature suggests MT-associated protein (MAP) $1 \mathrm{~B}$ as a substrate of GSK3 that controls MT dynamics in the growth cone (Goold et al. 1999; Trivedi et al. 2005). In addition to the configuration of growth cone MTs regulated by CLASP, other aspects, such as polymerization and dynamics of MTs, are also important parameters of axon growth. The possible interplays among CLASP and other GSK3 substrates to coordinate MT configuration and dynamics are yet to be determined.

\section{Plus end-binding activity of CLASP1/2 promotes axon regeneration}

MT stabilization is a prerequisite for axon regeneration (Erturk et al. 2007). Here we show that CLASP1/2 are required for rapid axon regeneration both in vitro and in vivo, and suggest that the plus end-binding activity of CLASP mediates this function by stabilizing MTs. Interestingly, compared with embryonic cortical neurons, the level of GSK3-Ser 9 phosphorylation was lower in adult sensory neurons, suggestive of higher GSK3 activity. However, the high intrinsic level of GSK3 activity observed in adult sensory neurons should be distinguished from aberrant activation of GSK3, as implicated in disease states (Medina and Castro 2008; Hernandez et al. 2009) or caused by overexpression of an active mutant of GSK3 (GSK3-S9A). Indeed, regenerative axon growth was substantially impaired by GSK3-S9A (Zhou et al. 2006), indicating that GSK3 inactivation to some extent is essential for axon regeneration. This observation is consistent with the fact that inhibition of GSK3 is required for the associations between MTs and several +TIPs, such as APC, ACF-7, and CLASP (Zhou et al. 2004; Kumar et al. 2009; Wu et al. 2011). It will be interesting to examine whether such +TIPs or other GSK3 substrates play a part in the regulation of axon regeneration after nerve injury.

Proposed model: GSK3-CLASP signaling as a regulator of axon growth rate

In epithelial cells, it has been shown that complete phosphorylation of all GSK3 sites in CLASP2 disrupts both the plus end-tracking and the lattice-binding properties, whereas partial phosphorylation of GSK3 sites specifically inhibits the lattice-binding activity of CLASP without altering its plus end-tracking property (Kumar et al. 2009). Thus, the different modes of CLASP-MT association observed in cortical and adult DRG neurons are likely the result of the different extent of CLASP phosphorylation that mirrors the level of GSK3 activity. When GSK3 activity is very high, most, if not all, GSK3 sites in CLASP are expected to be phosphorylated, detaching CLASP from both the plus ends and the lattices of MTs. Disruption of CLASPMT interaction leads to destabilization of axonal MTs, which will attenuate axon growth. As GSK3 activity becomes moderately inhibited, there may be dephosphorylation of some but not all GSK3 sites in CLASP, which allows CLASP to specifically associate with MT plus ends while still preventing its association along MT lattices. Preferential binding of CLASP to MT plus ends would protect the growing ends of MTs from destabilization and allow them to protrude into the actin-rich growth cone periphery, thereby facilitating axon extension. When GSK3 activity becomes strongly suppressed, as in the case of GSK3 knockdown, most, if not all, GSK3 sites are expected to be dephosphorylated, further increasing CLASP-MT association to the extent that CLASP binds along the distal region of MT lattices. Accumulation of CLASP along the lattices induces MT looping in the growth cone and restricts axon growth. Interestingly, we noticed a mobility shift in gels when we used lysates of cortical and adult DRG neurons: CLASP from adult DRG neuronal extracts migrated slower than that from cortical neuronal extracts, and treatment of adult DRG neuronal extracts with $\lambda$-phosphatase induced a downshift of CLASP /data not shown), consistent with the suggested different phosphorylation status of CLASP in the two types of neurons. However, further experiments are required to prove this model.

Unlike most other kinases, the conventional view is that GSK3 is highly active in cells under basal conditions and its inactivation is the switch to trigger downstream events. Previously, nerve growth factor (NGF) has been shown to promote axon growth through inactivation of GSK3, which employs APC to control neuronal MTs (Zhou et al. 2004). Thus, GSK3 inactivation has been proposed to enhance axon regeneration after spinal cord injuries (Dill et al. 2008). However, it should be noted that suppression of GSK3 activity to a greater extent blocks axon growth, indicating that activation of GSK3 is also crucial for efficient axon growth. The complete rescue of the axon growth defect observed in GSK3-depleted neurons by knocking down CLASP2, together with the structure-function analysis with various CLASP mutants, suggests that moderate activation of GSK3-which will specifically prevent CLASP from binding along MT lattices while keeping its association with the plus ends-is required for rapid axon extension. This model underscores GSK3 activation as an important aspect in the regulatory scheme, in addition to the currently well-established inactivation paradigm, and suggests that a delicate balance between activation and inactivation is required, highlighting the physiological imperative for appropriate regulation of GSK3 signaling. 
Given that GSK3 inhibition is suggested as a potential therapy in a variety of neurological diseases, identification of a major mediator of a certain neural defect and the mechanisms of action would be important not only in the field of basic science, but also in the field of translational research that targets GSK3.

\section{Materials and methods}

Antibodies, materials, and cDNA constructs

A full list of reagents, cDNA constructs, and antibodies is provided in the Supplemental Material (Supplemental Table S1).

\section{In vivo electroporation of adult DRG neurons}

All experiments involving animals were performed in accordance with the animal protocol approved by the Institutional Animal Care and Use Committee of the Johns Hopkins University. Under anesthesia induced by katamine $(100 \mathrm{mg} / \mathrm{kg})$ and xylazine $(10 \mathrm{mg} / \mathrm{kg})$, a skin incision was made and the overlaying muscles were removed from the L4-L5 spinous processes. A small dorsolateral laminectomy was then performed to expose the left L4-L5 DRGs. shCLASPs (or a control vector) were injected using pulled glass capillaries (Sutter Instrument) at $1.5 \mu \mathrm{L}$ per ganglion. Immediately after injection, electroporation was performed by applying five pulses (35 V, $10 \mathrm{msec}, 950-\mathrm{msec}$ interval) using a custom-made tweezer-like electrode $(\phi 1.2 \mathrm{~mm})$ with Electro Square Porator ECM830 (BTX Genetronics). The wound was then closed, and the mice were allowed to recover. Three days later, crush injuries of sciatic nerves were made with fine forceps, and the crushed sites were marked by 10-0 nylon epineural sutures. At $5 \mathrm{~d}$ after the sciatic nerve crush, the mice were perfused with $4 \%$ PFA in sodium phosphate buffer (pH 7.4). The whole nerve segment was dissected out and further fixed in $4 \%$ PFA overnight at $4^{\circ} \mathrm{C}$. Using the whole-mount nerve, the lengths of all identifiable Venus-labeled regenerating axons were measured from the crush site to the distal axon tips.

\section{Image analysis and statistics}

Axonal and dendritic lengths were measured with the "measure/ curve" application of AxioVision 4.6 software (Carl Zeiss MicroImaging, Inc.). For quantification of axon length, neurons with processes more than twice as long as one cell body diameter were photographed. The mean and SEM of neurite-bearing cells were calculated from at least three independent experiments. Images were cropped and/or enlarged to show representative examples in sufficient detail. To enable quantitative analysis of the MT structures in the growth cone, all free MT ends in the growth cone that could be identified were counted, and the number was normalized against the size of the growth cone. To quantify CLASP signal in the distal ends of axons in E15 cortical and regenerating adult DRG neurons, two neuronal samples were immunostained side by side. Total fluorescence intensity of CLASP immunostaining was measured in the growth cone or in the distal $\sim 20-\mu \mathrm{m}$ area when the growth cone morphology could not be completely distinguished from the axon. The intensity was normalized by the size of the selected area after subtracting the background fluorescence. To quantify CLASP-MT interactions in growth cones, we measured the lengths of CLASP immunostaining that displayed specific binding at the tip of MTs or along MT lattices. CLASP signal was determined by fluorescence intensity of immunostaining, and the length was measured until the fluorescence intensity dropped to the basal level. All data are pre- sented as mean \pm SEM from at least three independent experiments. Student's $t$-test was used to determine significance unless indicated otherwise.

\section{Supplemental material}

See the Supplemental Material for details of reagents used in the study and methods for cell culture, transfection, and immunofluorescence studies.

\section{Acknowledgments}

We are grateful to Drs. Anna Akhmanova and Torsten Wittmann for cDNA constructs, and to Drs. Irina Kaverina and Fedor Severin for CLASP antibodies. We thank Drs. Alex Kolodkin, Philip Nicovich, Woo Yang Kim, and Christopher Cohan for critical reading of the manuscript and insightful comments. This work was supported by the Christopher and Dana Reeve Foundation (E.M.H.), the Travis Roy Foundation (E.M.H.), the Whitehall Foundation (F.Q.Z.), the Basil O'Connor Starter Scholar Research Award of March of Dimes (F.Q.Z.), and NIH grant R01NS064288 (F.Q.Z.).

\section{References}

Akhmanova A, Steinmetz MO. 2008. Tracking the ends: a dynamic protein network controls the fate of microtubule tips. Nat Rev Mol Cell Biol 9: 309-322.

Akhmanova A, Hoogenraad CC, Drabek K, Stepanova T, Dortland B, Verkerk T, Vermeulen W, Burgering BM, De Zeeuw CI, Grosveld F, et al. 2001. Clasps are CLIP-115 and -170 associating proteins involved in the regional regulation of microtubule dynamics in motile fibroblasts. Cell 104: 923-935.

Al-Bassam J, Kim H, Brouhard G, van Oijen A, Harrison SC, Chang F. 2010. CLASP promotes microtubule rescue by recruiting tubulin dimers to the microtubule. Dev Cell 19: 245-258.

Allingham JS, Smith R, Rayment I. 2005. The structural basis of blebbistatin inhibition and specificity for myosin II. Nat Struct Mol Biol 12: 378-379.

Arimura N, Kaibuchi K. 2007. Neuronal polarity: from extracellular signals to intracellular mechanisms. Nat Rev Neurosci 8: 194-205.

Bratman SV, Chang F. 2008. Mechanisms for maintaining microtubule bundles. Trends Cell Biol 18: 580-586.

Burnette DT, Schaefer AW, Ji L, Danuser G, Forscher P. 2007. Filopodial actin bundles are not necessary for microtubule advance into the peripheral domain of Aplysia neuronal growth cones. Nat Cell Biol 9: 1360-1369.

Conde C, Caceres A. 2009. Microtubule assembly, organization and dynamics in axons and dendrites. Nat Rev Neurosci 10: 319-332.

Dill J, Wang H, Zhou F, Li S. 2008. Inactivation of glycogen synthase kinase 3 promotes axonal growth and recovery in the CNS. J Neurosci 28: 8914-8928.

Drabek K, van Ham M, Stepanova T, Draegestein K, van Horssen R, Sayas CL, Akhmanova A, Ten Hagen T, Smits $\mathrm{R}$, Fodde R, et al. 2006. Role of CLASP2 in microtubule stabilization and the regulation of persistent motility. Curr Biol 16: 2259-2264.

Erturk A, Hellal F, Enes J, Bradke F. 2007. Disorganized microtubules underlie the formation of retraction bulbs and the failure of axonal regeneration. J Neurosci 27: 9169-9180.

Even-Ram S, Doyle AD, Conti MA, Matsumoto K, Adelstein RS, Yamada KM. 2007. Myosin IIA regulates cell motility and actomyosin-microtubule crosstalk. Nat Cell Biol 9: 299-309. 
Frame S, Cohen P, Biondi RM. 2001. A common phosphate binding site explains the unique substrate specificity of GSK3 and its inactivation by phosphorylation. Mol Cell 7: 1321-1327.

Galjart N. 2005. CLIPs and CLASPs and cellular dynamics. Nat Rev Mol Cell Biol 6: 487-498.

Geraldo S, Khanzada UK, Parsons M, Chilton JK, Gordon-Weeks PR. 2008. Targeting of the F-actin-binding protein drebrin by the microtubule plus-tip protein EB3 is required for neuritogenesis. Nat Cell Biol 10: 1181-1189.

Goold RG, Owen R, Gordon-Weeks PR. 1999. Glycogen synthase kinase $3 \beta$ phosphorylation of microtubule-associated protein $1 \mathrm{~B}$ regulates the stability of microtubules in growth cones. J Cell Sci 112: 3373-3384.

Hernandez F, Nido JD, Avila J, Villanueva N. 2009. GSK3 inhibitors and disease. Mini Rev Med Chem 9: 1024-1029.

Hur EM, Zhou FQ. 2010. GSK3 signalling in neural development. Nat Rev Neurosci 11: 539-551.

Hur EM, Yang IH, Kim DH, Byun J, Saijilafu , Xu WL, Nicovich PR, Cheong R, Levchenko A, Thakor N, et al. 2011. Engineering neuronal growth cones to promote axon regeneration over inhibitory molecules. Proc Natl Acad Sci 108: 50575062.

Jiang H, Guo W, Liang X, Rao Y. 2005. Both the establishment and the maintenance of neuronal polarity require active mechanisms: critical roles of GSK-3 $\beta$ and its upstream regulators. Cell 120: 123-135.

Jope RS, Johnson GV. 2004. The glamour and gloom of glycogen synthase kinase-3. Trends Biochem Sci 29: 95-102.

Kim WY, Zhou FQ, Zhou J, Yokota Y, Wang YM, Yoshimura T, Kaibuchi K, Woodgett JR, Anton ES, Snider WD. 2006. Essential roles for GSK-3s and GSK-3-primed substrates in neurotrophin-induced and hippocampal axon growth. Neuron 52: 981-996.

Kumar P, Lyle KS, Gierke S, Matov A, Danuser G, Wittmann T. 2009. GSK3 $\beta$ phosphorylation modulates CLASP-microtubule association and lamella microtubule attachment. J Cell Biol 184: 895-908.

Lee H, Engel U, Rusch J, Scherrer S, Sheard K, Van Vactor D. 2004. The microtubule plus end tracking protein Orbit/ MAST/CLASP acts downstream of the tyrosine kinase Abl in mediating axon guidance. Neuron 42: 913-926.

Lowery LA, Lee H, Lu C, Murphy R, Obar RA, Zhai B, Schedl M, Van Vactor D, Zhan Y. 2010. Parallel genetic and proteomic screens identify Msps as a CLASP-Abl pathway interactor in Drosophila. Genetics 185: 1311-1325.

Martinez A. 2008. Preclinical efficacy on GSK-3 inhibitors: towards a future generation of powerful drugs. Med Res Rev 28: 773-796.

Medina M, Castro A. 2008. Glycogen synthase kinase-3 (GSK-3) inhibitors reach the clinic. Curr Opin Drug Discov Devel 11: 533-543.

Mimori-Kiyosue Y, Grigoriev I, Lansbergen G, Sasaki H, Matsui C, Severin F, Galjart N, Grosveld F, Vorobjev I, Tsukita S, et al. 2005. CLASP1 and CLASP2 bind to EB1 and regulate microtubule plus-end dynamics at the cell cortex. I Cell Biol 168: 141-153.

Neukirchen D, Bradke F. 2011. Cytoplasmic linker proteins regulate neuronal polarization through microtubule and growth cone dynamics. J Neurosci 31: 1528-1538.

Owen R, Gordon-Weeks PR. 2003. Inhibition of glycogen synthase kinase $3 \beta$ in sensory neurons in culture alters filopodia dynamics and microtubule distribution in growth cones. Mol Cell Neurosci 23: 626-637.

Phukan S, Babu VS, Kannoji A, Hariharan R, Balaji VN. 2010. GSK3 $\beta$ : role in therapeutic landscape and development of modulators. Br J Pharmacol 160: 1-19.
Schaefer AW, Kabir N, Forscher P. 2002. Filopodia and actin arcs guide the assembly and transport of two populations of microtubules with unique dynamic parameters in neuronal growth cones. J Cell Biol 158: 139-152.

Siegrist SE, Doe CQ. 2007. Microtubule-induced cortical cell polarity. Genes Dev 21: 483-496.

Stiess M, Bradke F. 2011. Neuronal polarization: the cytoskeleton leads the way. Dev Neurobiol 71: 430-444.

Straight AF, Cheung A, Limouze I, Chen I, Westwood NJ, Sellers JR, Mitchison TJ. 2003. Dissecting temporal and spatial control of cytokinesis with a myosin II Inhibitor. Science 299: 1743-1747.

Trivedi N, Marsh P, Goold RG, Wood-Kaczmar A, GordonWeeks PR. 2005. Glycogen synthase kinase- $3 \beta$ phosphorylation of MAP1B at Ser1260 and Thr1265 is spatially restricted to growing axons. I Cell Sci 118: 993-1005.

Tsvetkov AS, Samsonov A, Akhmanova A, Galjart N, Popov SV. 2007. Microtubule-binding proteins CLASP1 and CLASP2 interact with actin filaments. Cell Motil Cytoskeleton 64: 519-530.

Watanabe T, Noritake J, Kakeno M, Matsui T, Harada T, Wang S, Itoh N, Sato K, Matsuzawa K, Iwamatsu A, et al. 2009. Phosphorylation of CLASP2 by GSK-3 $\beta$ regulates its interaction with IQGAP1, EB1 and microtubules. I Cell Sci 122: 2969-2979.

Wittmann T, Waterman-Storer CM. 2005. Spatial regulation of CLASP affinity for microtubules by Rac1 and GSK3 $\beta$ in migrating epithelial cells. J Cell Biol 169: 929-939.

Wu X, Shen QT, Oristian DS, Lu CP, Zheng Q, Wang HW, Fuchs E. 2011. Skin stem cells orchestrate directional migration by regulating microtubule-ACF7 connections through GSK3 3 . Cell 144: 341-352.

Yoshimura T, Kawano Y, Arimura N, Kawabata S, Kikuchi A, Kaibuchi K. 2005. GSK-3 $\beta$ regulates phosphorylation of CRMP-2 and neuronal polarity. Cell 120: 137-149.

Zhou FQ, Zhou J, Dedhar S, Wu YH, Snider WD. 2004. NGFinduced axon growth is mediated by localized inactivation of GSK-3 $\beta$ and functions of the microtubule plus end binding protein APC. Neuron 42: 897-912.

Zhou FQ, Walzer M, Wu YH, Zhou J, Dedhar S, Snider WD. 2006. Neurotrophins support regenerative axon assembly over CSPGs by an ECM-integrin-independent mechanism. J Cell Sci 119: 2787-2796. 


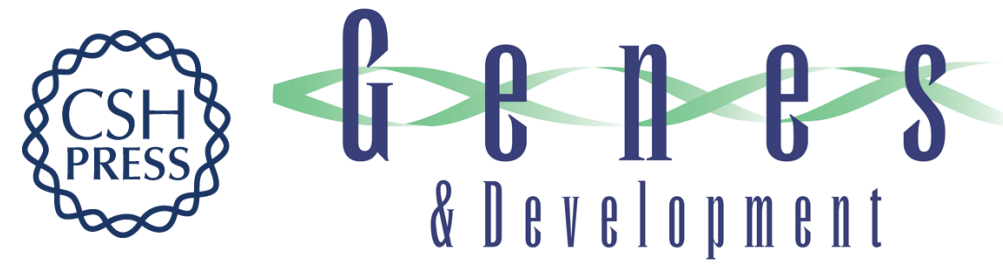

\section{GSK3 controls axon growth via CLASP-mediated regulation of growth cone microtubules}

Eun-Mi Hur, Saijilafu, Byoung Dae Lee, et al.

Genes Dev. 2011, 25:

Access the most recent version at doi:10.1101/gad.17015911

Supplemental http://genesdev.cshlp.org/content/suppl/2011/09/21/25.18.1968.DC1
Material

References This article cites 43 articles, 14 of which can be accessed free at: http://genesdev.cshlp.org/content/25/18/1968.full.html\#ref-list-1

License

Email Alerting Receive free email alerts when new articles cite this article - sign up in the box at the top Service right corner of the article or click here.

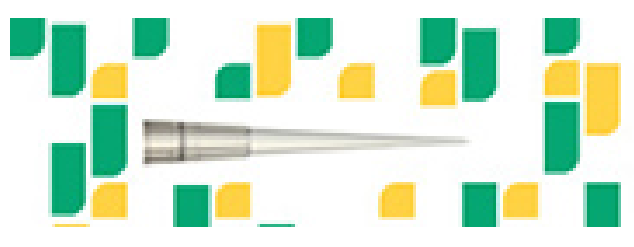

Focused on your science. 